Mens

revue d'histoire intellectuelle de l'Amérique française

\title{
Usage du passé et mémoire collective franco-ontarienne : le souvenir du Règlement 17 dans la bataille pour sauver l'hôpital Montfort
}

\section{Marcel Martel}

Volume 6, numéro 1, automne 2005

URI : https://id.erudit.org/iderudit/1024256ar

DOI : https://doi.org/10.7202/1024256ar

Aller au sommaire du numéro

Éditeur(s)

Centre de recherche en civilisation canadienne-française

ISSN

1492-8647 (imprimé)

1927-9299 (numérique)

Découvrir la revue

Citer cet article

Martel, M. (2005). Usage du passé et mémoire collective franco-ontarienne : le souvenir du Règlement 17 dans la bataille pour sauver l'hôpital Montfort.

Mens, 6(1), 69-94. https://doi.org/10.7202/1024256ar
Résumé de l'article

C'est en se référant à la lutte contre le Règlement 17 que la présidente du comité S.O.S. Montfort, Gisèle Lalonde, présente la lutte pour sauver l'hôpital Montfort. Cet épisode constitue un cas d'analyse des efforts, déployés par les responsables de S.O.S. Montfort, pour mobiliser la communauté en se référant à l'histoire. C'est aussi l'occasion d'analyser les usages précis de la mémoire collective des Franco-Ontariens dans le but de donner du sens à l'expérience historique de la communauté. 


\title{
USAGE DU PASSÉ \\ ET MÉMOIRE COLLECTIVE \\ FRANCO-ONTARIENNE : \\ LE SOUVENIR DU RËLEMENT 17 \\ DANS LA BATAILLE POUR SAUVER \\ L'HÔPITAL MONTFORT' ${ }^{1}$
}

\author{
Marcel Martel \\ Département d'histoire \\ York University
}

\section{Résumé}

C'est en se référant à la lutte contre le Règlement 17 que la présidente du comité S.O.S. Montfort, Gisèle Lalonde, présente la lutte pour sauver l'hôpital Montfort. Cet épisode constitue un cas d'analyse des efforts, déployés par les responsables de S.O.S. Montfort, pour mobiliser la communauté en se référant à l'histoire. C'est aussi l'occasion d'analyser les usages précis de la mémoire collective des Franco-Ontariens dans le but de donner du sens à l'expérience historique de la communauté.

\section{Abstract}

Gisele Lalonde, the leader of S.O.S. Montfort, explained the struggle to save Ottawa's Montfort hospital by constant references to the early twentieth-century struggle against Ontario's infamous Regulation 17. The Montfort issue, therefore, provides an opportunity to analyse bow the coalition to save the hospital used bistory in order to mobilize the Franco-Ontarian community. Furthermore, an analysis of the struggle to save Montfort reveals which specific references to the Franco-Ontarian collective memory were used in order to explain the history of this community. 
«La bataille qui s'engage se compare à celle menée avec succès contre le Règlement $17^{2} »$. C'est avec une telle référence à l'histoire que Gisèle Lalonde, présidente du comité S.O.S. Montfort, présente la lutte pour garder l'hôpital Montfort dès le lendemain de l'annonce de la recommandation de la fermeture de cet établissement hospitalier par la Commission de restructuration des services de santé en Ontario, le 24 février 1997. Dans les jours qui ont suivi l'annonce de la fermeture de l'hôpital Montfort, le Règlement 17, événement devenu un mythe fondateur de l'identité de la communauté franco-ontarienne, est constamment évoqué par ceux et celles qui tentent d'infléchir la décision des membres de la Commission et, indirectement, celle du gouvernement conservateur de Mike Harris. Le rappel de cet événement historique n'est toutefois pas anodin. Il témoigne d'abord d'une volonté de mobilisation, mobilisation qui, de l'avis des instigateurs, sera nécessairement victorieuse à l'instar de la bataille contre le Règlement 17. Il évoque aussi le désir d'inscrire le combat pour sauver l'hôpital Montfort dans la continuité de l'histoire de la communauté franco-ontarienne. Bref, la lutte pour sauver l'hôpital Montfort nous permet d'entrer dans l'imaginaire symbolique de la communauté et d'identifier l'usage du passé à des fins politiques. Cet article explore la mémoire collective des Franco-Ontariens à un moment précis de leur histoire ou, pour être plus précis, examine le contenu d'une mémoire véhiculée par un groupe influent de cette communauté, principalement les dirigeants de la coalition S.O.S. Montfort. Ce groupe est tellement influent qu'il se présente et se définit comme le représentant et le défenseur de la communauté.

Aborder la question de la mémoire oblige à nous interroger sur son contenu. Qu'entendons-nous par mémoire ? Pour reprendre les propos de Béatrice Richard, il s'agit de la mémoire «comme fonction organisatrice du récit collectif». Ce 
récit collectif est biaisé puisqu'il est soumis « aux manipulations conscientes ou inconscientes que l'intérêt, l'affectivité, le désir, l'inhibition, la censure, exercent sur la mémoire individuelle ${ }^{3} \%$.

Mon étude s'inscrit dans un courant historiographique qui gagne en popularité, soit l'étude de l'usage du passé par une société, mais plus souvent qu'à leur tour par les élites politiques et intellectuelles, dans le cadre d'une commémoration comme l'a révélé l'étude stimulante de Henry Vivian Nelles sur les préparatifs et la mise en forme des festivités du tricentenaire de Québec en 1908, ou encore dans la course à l'érection de plaques historiques comme l'atteste l'étude d'Alan Gordon ou enfin la déformation du passé dans la création du " héros » Dollard des Ormeaux qui, selon Patrice Groulx, peut expliquer les difficultés dans le dialogue et les relations entre les Québécois francophones et les nations amérindiennes ${ }^{4}$. Ces études démontrent, comme l'a souligné Sacha Richard, que " la commémoration se répercute sur la (re)création d'identités collectives, car elle redéfinit la relation d'une collectivité à son passé5 $»$.

Le cas de la bataille pour sauver l'hôpital Montfort ainsi que la mobilisation d'éléments et de symboles de la mémoire collective des Franco-Ontariens démontrent qu'il s'agit, entre autres, d'une "strategy against the oppression, assimilation, and extinction of community identity ${ }^{6} »$. Il s'agit ici d'un groupe minoritaire, dont la capacité d'action sur l'État est limitée, qui amorce, malgré tout, une lutte pour sauver une institution. Dans cette lutte qui puise notamment dans la mémoire collective des Franco-Ontariens, il y a une volonté de déformation des faits, mais une déformation qui obéit à une stratégie de l'usage du passé, destinée à fournir « un certain nombre de clés pour la compréhension du présent ${ }^{7}$ ». Puisque l'épisode de la crise du Règlement 17 joue un rôle 
dans la bataille pour sauver l'hôpital Montfort, cet article porte d'abord sur les motivations qui incitent les dirigeants de la coalition à se référer à cet épisode historique. Ensuite, il passe en revue les causes de ces crises respectives ainsi que la mobilisation à laquelle ces événements ont donné lieu. Enfin, il s'intéresse aux faits saillants de la mémoire collective auxquels font appel les dirigeants de la coalition S.O.S. Montfort.

\section{Un usage précis d'un événement historique : la référence au Règlement 17}

Durant la période intense de mobilisation qui précède le rassemblement du 22 mars 1997 au Centre municipal d'Ottawa, nous assistons à une " étonnante effervescence mythologique ", pour reprendre les propos de Raoul Girardet dans son étude sur les mythes en politique, puisque les références au Règlement 17 abondent. Le quotidien Le Droit affirme que cette directive gouvernementale en matière d'éducation et l'hôpital Montfort sont des combats similaires. Dans son éditorial, intitulé " La gifle», Pierre Bergeron écrit :

Le Droit fut fondé le 27 mars 1913 pour lutter contre le Règlement 17 concocté par les forces obscurantistes de l'écrasement et de l'assimilation des francophones de l'Ontario. Lundi, leurs héritiers ont essuyé une autre gifle de même nature avec l'annonce de la fermeture de l'hôpital Montfort dans le cadre de la restructuration des services de santé dans Ottawa-Carleton?.

Lors du rassemblement du 22 mars, auquel assistent plus de 10000 personnes, plusieurs des conférenciers évoquent le Règlement 17 dans des discours parfois enflammés. Ainsi, Denis Pommainville, représentant du Conseil régional pour la santé mentale de Prescott-Russell, n'hésite pas à proposer un raccourci historique en présentant le cas Montfort comme la répétition, quatre-vingts ans plus tard, de la lutte contre cette 
directive gouvernementale. "Si Mike Harris croyait que les Franco-Ontariens allaient perdre leur mémoire collective, il s'est trompé. [...] Nous nous souvenons du 22 mars 1911, la date du Règlement $17^{10}$ ». Dans le cahier spécial publié par Le Droit au lendemain du rassemblement du 22 mars, la publicité de l'Association canadienne-française de l'Ontario (ACFO) et de ses ACFO régionales est révélatrice de la place particulière du Règlement 17. Dans cette publicité, l'ACFO signale une série d'événements, tels que l'abrogation du Règlement 17 en 1927, la loi fédérale sur les langues officielles de 1969 ou encore la loi ontarienne sur les services en français de 1986, qui constituent des gains pour la communauté puisqu'ils ont favorisé l'avancement des droits des FrancoOntariens. Ces faits deviennent des révélateurs de la conscience historique franco-ontarienne ou encore des repères dans le temps pour marquer l'enracinement et la progression dans la reconnaissance des droits de la communauté en Ontario et au Canada. Le Règlement 17 est toutefois le seul événement mentionné avant l'adoption de la loi sur les langues officielles en $1969^{11}$. Il est intéressant de relever qu'il s'agit surtout d'événements de nature législative, comme si une communauté ne pouvait s'affirmer que dans les moments où celle-ci marque des gains de cet ordre. C'est révélateur de la place des lois dans la culture des groupes minoritaires mais aussi chez la population canadienne. Comme le rappelle le politologue Alan Cairns, la Charte des droits et libertés de la personne a modifié les paramètres de la culture politique et surtout la définition du nous canadien ${ }^{12}$. Ainsi, la Charte des droits de la personne a marqué l'imaginaire des Canadiens et conséquemment les communautés minoritaires, leur perception du pays et leur représentation de la collectivité.

Dans son autobiographie, la dirigeante de la coalition pour sauver l'hôpital Montfort explique les raisons du choix 
de la référence au Règlement 17. Dans les heures qui ont suivi son acceptation de diriger la coalition, Gisèle Lalonde travaille sur sa déclaration, qui sera lue lors de la conférence de presse prévue le lendemain de l'annonce de la Commission de restructuration des soins de santé. Elle est à la recherche de mots "qui sauront rallier toute la communauté ${ }^{13}$ ", mais aussi injecteront une bonne dose d'optimisme au sein des troupes dont il faut fouetter l'ardeur et surtout grossir les rangs. En effet, Gisèle Lalonde sait que la bataille sera ardue et frustrante pour les Franco-Ontariens, puisqu'il faut convaincre un gouvernement déterminé à aller de l'avant avec sa " révolution du bon sens » et son programme de coupures de service et de réduction de la taille de l'État. Elle se consacre à cette délicate tâche et en arrive à établir un lien avec la crise scolaire puisqu'il s'impose : «je veux immédiatement comparer cela au Règlement 17 (1912), l'événement le plus tragique de notre histoire franco-ontarienne ${ }^{14} \%$.

Il peut paraitre surprenant que celle qui dirige la campagne pour sauver Montfort fasse appel à l'histoire pour donner un sens à l'événement qui vient perturber la communauté franco-ontarienne, pour présenter les enjeux de la bataille qui s'annonce, ainsi que pour susciter la mobilisation. Il est intéressant que Clio, la muse de l'histoire, devienne une conseillère des dirigeants de S.O.S. Montfort et notamment de sa présidente. Ce n'est donc pas une bataille contre le néo-libéralisme, qui anime les dirigeants de S.O.S. Montfort. Il s'agit plutôt d'une bataille, résultant d'une décision, prise par une composante étatique. Cette bataille illustre les relations entre les Franco-Ontariens et l'État provincial ontarien, qui, à l'occasion, deviennent conflictuelles lorsque des composantes étatiques posent des gestes nuisant au développement de la collectivité franco-ontarienne en général, et plus particulièrement de celle de la capitale nationale et de l'Est ontarien, dans le 
cas de la décision de la Commission de restructuration des services de santé en Ontario.

En se référant au Règlement 17, Gisèle Lalonde a plusieurs objectifs en tête. Le premier est sans contredit celui d'ancrer l'événement Montfort dans l'histoire de la communauté franco-ontarienne. Le personnel de l'hôpital, les patients et la communauté subissent un affront. Mais ils ne sont pas les seuls à le subir. En effet, c'est un affront à l'ensemble de la communauté, puisque l'hôpital Montfort est une institution servant la communauté. Pour Lalonde, Montfort est « une des grandes réussites du peuple franco-ontarien ${ }^{15} »$. Rappelons que la communauté religieuse des Filles de la Sagesse participe à la fondation de l'hôpital. Cette communauté en assure la gestion administrative, de 1953 jusqu'à ce que l'État ontarien prenne la relève en 1970. Lors de l'annonce de sa fermeture, l'hôpital compte alors 242 lits $^{16}$. Cette réussite du peuple franco-ontarien, pour reprendre les mots de Lalonde, démontre que Montfort est une institution qui a un rôle particulier, dépassant celui d'offrir des soins de santé. Puisque des membres de la communauté ont fondé cet établissement hospitalier, une attaque contre cette institution est construite, par Lalonde et les autres membres de la coalition, comme une attaque contre la communauté. Soucieux d'identifier des lieux de mémoire pour la communauté, la coalition S.O.S. Montfort fait de cet établissement hospitalier un lieu de mémoire en raison du symbole qu'elle lui confère ${ }^{17}$.

Par sa référence au Règlement 17, Gisèle Lalonde cherche aussi à élargir les rangs de la communauté, affectée par la décision de fermer l'hôpital Montfort. Tout comme le Règlement 17 a suscité une mobilisation qui a dépassé les frontières ontariennes, Lalonde cherche à faire de Montfort un enjeu national. En transformant la bataille locale en une lutte nationale pour le respect des droits des Franco-Ontariens, elle 
cherche à gagner des alliés. Certes, des considérations stratégiques interviennent, puisque le cas Montfort survient deux ans à peine après la tenue du référendum sur l'avenir politique du Québec en 1995. En fermant Montfort, on affirme que cette décision favorise la cause de ceux qui croient que hors du Québec, il n'y a pas de salut pour le fait français. Audelà de ces considérations stratégiques, en établissant un lien avec la crise du Règlement 17, Lalonde veut démontrer que la bataille qui s'amorce est similaire à celle survenue en 1912: c'est une bataille nationale. Devant les membres du Club Richelieu le 5 mars 1997, elle lance cet avertissement : «[...] le sort de l'Hôpital Montfort est une question qui devrait préoccuper tous les Canadiens et toutes les Canadiennes qui tiennent à l'unité du pays. L'avenir du Canada, tel que nous le connaissons et nous l'aimons, pourrait très bien se jouer ici ${ }^{18}$ ".

La référence à la crise du Règlement 17 atteste également d'une volonté de puiser dans l'imaginaire de la communauté franco-ontarienne en lui rappelant que la bataille pour l'établissement hospitalier s'inscrit dans les innombrables luttes qui ponctuent l'expérience historique des FrancoOntariens au vingtième siècle. Avec Montfort, cette lutte ouvre un nouveau front, celui de l'offre de soins de santé en français. Lorsque Gisèle Lalonde se réfère au Règlement 17, elle force les gens à retourner dans le passé. «Nos leaders et aînés francophones comprennent le sens de mes paroles. Mais les pauvres et jeunes journalistes, anglophones et francophones, veulent savoir ce qu'était le Règlement 17 et bien comprendre l'histoire des épingles à chapeau ${ }^{19} \%$. Il y a toutefois un pari : celui d'établir un lien avec un événement dont le souvenir est ambivalent. En effet, le Règlement 17 évoque un épisode douloureux pour la communauté puisque la crise a duré plus de 15 ans, soit de 1912 à 1927. Mais c'est aussi évoquer un épisode heureux, car la bataille a été gagnée et 
des héros et des héroïnes peuplent depuis le panthéon de la mémoire collective franco-ontarienne. Gisèle Lalonde en est consciente, comme elle le mentionne dans son autobiographie : "Je pense à Jeanne Lajoie, aux femmes de l'école Guigues avec leurs épingles à chapeaux. Elles en avaient eu, du courage ${ }^{20} »$. Donc, il y a une volonté d'ancrer la bataille dans le passé de la communauté pour y puiser l'énergie de combattre. Cette lutte requiert cependant une importante participation de la part de la communauté.

Les dirigeants de S.O.S. Montfort ont tôt fait d'interpréter la décision de la Commission comme une attaque. Le rappel d'une bataille pour sauvegarder une autre institution - l'école - constitue un choix, bien que le contexte historique dans lequel s'est déroulée la crise scolaire soit tout à fait différent.

\section{Montfort et le Règlement 17 : causes et mobilisation}

Lorsqu'en février 1997, la Commission de restructuration des services de santé en Ontario recommande la fermeture de l'hôpital Montfort, hôpital désigné pour offrir des services en français dans la région d'Ottawa et de l'Est ontarien, les milieux franco-ontariens réagissent avec stupeur. Ces derniers ont du mal à comprendre la logique de la décision de la Commission, car, croient-ils, le fait français est considéré comme un fait établi et respecté. Ainsi pour bon nombre de Franco-Ontariens, la "révolution du bon sens » - slogan politique de l'équipe conservatrice de Mike Harris durant la campagne électorale de 1995 — perd de son « bon sens » lorsqu'ils tentent de comprendre la recommandation de la Commission.

La politique de restriction budgétaire peut constituer un phare pour ceux qui scrutent l'horizon dans l'espoir d'y voir clair. Au nom de la lutte au déficit provincial, la politique 
gouvernementale oblige à restructurer les services de santé. Cette restructuration entraîne une diminution du nombre d'établissements de soins de santé dans la province. À Ottawa, la politique de restriction budgétaire, dictée par l'idéologie néolibérale, devient implacable. Elle entraîne la fermeture de trois hôpitaux, dont Montfort. Avec sa recommandation, la Commission de restructuration attaque une composante du réseau institutionnel franco-ontarien, réseau qui a lentement et patiemment été mis en place par les francophones, depuis leur arrivée en Ontario. Avec sa proposition de fermer une composante du réseau institutionnel régional, la Commission menace les stratégies élaborées pour assurer le maintien du fait français dans cette partie de l'Ontario. Le journaliste Charles Burroughs le souligne d'ailleurs lorsqu'il écrit que « jamais, depuis l'époque du Règlement 17, nous sommes-nous levés, en bloc, pour défier un gouvernement, populaire par surcroit, et clamer à pleins poumons que nos institutions francophones ne sont pas près de disparaittre ${ }^{21}$ !»

Lorsqu'il publie les restrictions imposées à l'enseignement en français dans les écoles de la province, en juin 1912, le gouvernement conservateur de James Whitney est influencé par le courant d'hostilité à l'égard du fait français et du catholicisme qui secoue le pays depuis les années 1880. Cette hostilité s'explique en partie par l'arrivée de milliers d'immigrants au pays. Entre 1890 et 1914, plus de trois millions d'entre eux entrent au pays, parmi lesquels un bon nombre ne proviennent pas des îles britanniques ${ }^{22}$. Devant l'afflux de ces milliers d'immigrants aux pratiques culturelles différentes de celles des Britanniques, les élites anglophones craignent pour le caractère anglo-saxon et protestant du pays. Ce contexte de peur et de lutte pour l'affirmation du fait britannique protestant justifie l'abolition du français comme langue officielle au Manitoba et la fin du financement des écoles séparées par 
l'État provincial, en 1890. Dorénavant l'école manitobaine, qui était un outil de reproduction culturelle, devient un instrument destiné à assimiler les enfants ${ }^{23}$.

Une peur similaire anime les élites protestantes anglosaxonnes qui, en Ontario, estiment que leur province est envahie. Ces gens craignent pour le maintien des institutions britanniques et protestantes nécessaires à l'indépendance du Canada comme nation distincte du géant américain. Ces élites tolèrent le fait français, à condition qu'il soit contenu au Québec. Lorsque des Canadiens français traversent en grand nombre la frontière québécoise pour venir s'installer dans l'Est ontarien ou dans le territoire du Nouvel-Ontario, la peur de l'envahissement vient hanter ces élites déjà inquiètes. D'ailleurs, en moins de trente ans, la population canadiennefrançaise double en Ontario. De 1881 à 1911, les Canadiens français passent de 102473 à $202442^{24}$. Il s'agit, pour reprendre l'expression de l'historien Pierre Savard, d'une « invasion silencieuse " par les Canadiens français ${ }^{25}$.

En 1905, les partisans de l'anglais comme unique langue commune de la province portent au pouvoir un parti politique, l'équipe conservatrice, sensible à leurs inquiétudes. En 1910, le gouvernement Whitney demande au $\mathrm{D}^{\mathrm{r}} \mathrm{F}$. W. Merchant d'enquêter sur les écoles dites bilingues. Dans son rapport, présenté deux ans plus tard à l'Assemblée législative, le $\mathrm{D}^{\mathrm{r}}$ Merchant signale quelques faiblesses dans les écoles bilingues, fréquentées surtout par les jeunes canadiens-français. Le manque d'efficacité de ces écoles et le fait que les enfants quittent l'établissement scolaire avec un bagage insuffisant inquiètent l'enquêteur. Le remède consiste à accroittre la part de l'enseignement de l'anglais ${ }^{26}$. Pour le gouvernement, une solution beaucoup plus radicale s'impose. Par la publication du Règlement 17, le ministère de l'Éducation limite l'enseignement du français aux deux premières années du primaire 
dans les écoles publiques et séparées. Le français est interdit comme langue d'enseignement au-delà de la deuxième année.

Le Règlement 17 réjouit les partisans de l'anglais comme unique langue commune. Les rangs de ces derniers comptent bien entendu les diverses loges orangistes, mais aussi certains membres du clergé catholique dont plusieurs d'entre eux sont d'origine irlandaise. Ces derniers ont encouragé le gouvernement à interdire l'enseignement dans une langue autre que l'anglais. Le nom de $\mathrm{M}^{\mathrm{gr}}$ Michael Francis Fallon, ancien professeur de littérature anglaise à l'Université d'Ottawa devenu évêque de London, en 1910, est associé à cette lutte acharnée en faveur de l'anglais comme unique langue d'enseignement. $\mathrm{M}^{\mathrm{gt}}$ Fallon en fait d'ailleurs part au ministre ontarien de l'Éducation dans sa lettre du 2 janvier 1912 $2^{27}$. Pour $\mathrm{M}^{\mathrm{gr}}$ Fallon, il importe de sauver les écoles séparées, de peur que tous les catholiques ne subissent la furie des élites anglo-protestantes comme ce fut le cas au Manitoba.

Quatre-vingt-cinq ans avant Montfort, la communauté canadienne-française de l'Ontario a été interpellée par l'action gouvernementale. Comme l'a démontré Chad Gaffield dans son étude sur le comté de Prescott, les communautés canadiennes-françaises font de l'école un instrument qui leur permet d'assurer la transmission de leur bagage culturel, et ce, au moment où les enfants ne constituent plus une source de revenu indispensable dans les stratégies familiales de survie économique. Le Règlement 17 vient cependant menacer la fonction de transmission du bagage culturel ${ }^{28}$.

Ainsi, les origines des deux crises diffèrent. En 1912, les inquiétudes des élites anglophones et de certains membres du clergé irlandais à propos de la survie du caractère protestant et britannique de la province amènent le gouvernement, qui partage ces inquiétudes, à imposer des restrictions à l'enseignement en français. En 1997, l'affaire de l'hô- 
pital Montfort fait suite à l'adoption d'une politique budgétaire restrictive s'inscrivant dans le cadre du néo-libéralisme basé sur la réduction de la taille de l'État, de la baisse des taxes et impôts personnels. Dans les deux cas, cependant, l'existence des institutions jugées indispensables au maintien de la communauté est mise en cause. L'importance de ces institutions dans la stratégie de défense et de promotion des droits des Franco-Ontariens explique la mobilisation pour les sauvegarder.

Tant au moment de la longue résistance au Règlement 17, de 1912 à 1927, qu'à celui de la lutte pour le maintien de l'hôpital Montfort, la mobilisation des francophones est importante. Dans le cas de Montfort, les bénéficiaires des soins de santé, le personnel et les fondatrices de l'hôpital occupent un rôle de premier plan. Lors de la bataille du Règlement 17, les enseignantes, dont Jeanne Lajoie de Pembrooke, qui a été congédiée en 1923, les parents et les enfants, sont aux premiers rangs de la lutte pour la sauvegarde des écoles. La manifestation en faveur de l'école Guigues, en 1916, au cours de laquelle les femmes ont pris la défense de cet établissement, armées de leurs épingles à chapeau, a marqué l'imaginaire. D'ailleurs, Gisèle Lalonde n'hésite pas à en rappeler le souve$\mathrm{nir}^{29}$.

Les dirigeants de l'Association canadienne-française d'éducation de l'Ontario (ACFEO), qui vient tout juste d'être fondée en 1910, cherchent à multiplier leurs alliés. La création du quotidien Le Droit, en 1912, permet de doter la communauté d'un outil de combat qui assure une couverture médiatique de la résistance au Règlement 17. Lors de la bataille pour l'hôpital Montfort, Le Droit appuie résolument cette cause. Cet appui se justifie, selon l'éditeur Pierre Bergeron, par le fait que le quotidien "renou[e] avec ses racines et réveill[e] le souvenir des luttes historiques ${ }^{30} »$. 
Le clergé canadien-français intervient aux côtés des parents et des dirigeants de l'ACFEO dans la crise scolaire. L'Église catholique est toutefois divisée, ce qui oblige Rome à rappeler les évêques à l'ordre. À plusieurs reprises, les évêques canadiens-français prient le Vatican d'appuyer les FrancoOntariens. Dans sa lettre collective au pape Benoît XV en date du 15 juin 1915, l'épiscopat canadien-français rappelle que la lutte des Canadiens français est tout à fait légitime et importante pour le devenir des écoles confessionnelles en Ontario mais aussi au Québec. Pour leur part, les évêques irlandais font entendre un tout autre son de cloche. Ils soulignent aux autorités du Vatican que l'anglais est la langue de la majorité en Ontario. Une maitrise imparfaite de la langue de la majorité condamne les enfants canadiens-français à un " état d'infériorité socio-économique ${ }^{31}$ ».

Le Vatican envoie deux lettres afin de rétablir la concorde. La première, la lettre encyclique Commissio divinitus en date du 18 septembre 1916, dénonce la division au sein de l'Église canadienne, reconnaît au gouvernement le droit d'exiger l'enseignement de l'anglais et permet aux Canadiens français «de réclamer, quoique dans une proportion convenable, que dans les écoles, où leurs enfants sont en un certain nombre, la langue française soit enseignée ${ }^{32} \%$. Les évêques canadiens-français espéraient un appui inconditionnel de Rome, ce qu'ils n'ont pas obtenu puisque la lettre prie les évêques, déjà divisés entre eux, de trouver une solution à la crise. Deux ans plus tard, soit le 7 juin 1918, une nouvelle lettre est envoyée aux évêques canadiens. Intitulée Litteris apostolicis, ce document ordonne le retour de la paix au sein de l'Église canadienne $\mathrm{e}^{33}$.

Ces querelles et divisions au sein de l'Église contrastent avec ce que l'on observe dans le cas de l'hôpital Montfort. D'abord, depuis la fin du concile Vatican II, l'Église n'est 
plus le maitre d'œuvre de l'organisation du social et du réseau institutionnel ni en Ontario français, ni au Québec. En dépit de cela, l'appui de l'Église est acquis aux défenseurs de Montfort. Dans les jours qui ont suivi l'annonce de la fermeture, le curé Jean-Pierre Fredette de la paroisse Saint-Joseph d'Orléans fait circuler une pétition, geste imité par d'autres prêtres du diocèse ${ }^{34}$. La présence de l'évêque auxiliaire d'Ottawa, $\mathrm{M}^{\mathrm{gt}}$ Paul Marchand, au rassemblement du 22 mars, témoigne de l'appui de l'Église ${ }^{35}$.

Le cas de l'hôpital Montfort interpelle les politiciens québécois. Le premier ministre du Québec Lucien Bouchard et le chef de l'opposition libérale, Daniel Johnson, se prononcent en faveur de Montfort. Lors d'une rencontre avec son homologue ontarien le 7 mars, le premier ministre Bouchard se livre, selon une dépêche de la Presse canadienne, à un "vibrant plaidoyer ${ }^{36} »$. Quelques jours auparavant, le premier ministre Bouchard avait déclaré son appui à la cause de l'hôpital Montfort: "Pour moi, il est inimaginable qu'il soit fermé ${ }^{37} »$.

Des gestes similaires sont posés de la part des politiciens du Québec lors de la crise scolaire du début du vingtième siècle. En janvier 1915, le premier ministre Lomer Gouin demande à l'Ontario de faire preuve de générosité à l'égard des Canadiens français. L'Assemblée législative du Québec adopte une motion enjoignant l'Ontario de respecter les droits des Canadiens français ${ }^{38}$. Plus tard, le premier ministre Alexandre Taschereau exercera une influence discrète sur son homologue ontarien, G. Howard Ferguson, en lui rappelant que le dossier du Règlement 17 gagnerait à être réglé. Ce geste de Taschereau s'explique par le fait que, dans les années 1920, le gouvernement du Québec est désireux de vendre de l'électricité à cette province ${ }^{39}$. 
Les politiciens fédéraux ne demeurent pas indifférents aux deux crises. Dans le cas de Montfort, le premier ministre Jean Chrétien manifeste son appui, après le " vibrant plaidoyer " du premier ministre Bouchard. Jusqu'à sa déclaration, le silence de Chrétien en agaçait plusieurs parmi les dirigeants de la coalition S.O.S. Montfort. L'éditeur du Droit, Pierre Bergeron, a même critiqué le comportement du premier ministre canadien en écrivant que le chef du gouvernement «ne [pouvait] moralement se rebattre derrière l'argument de la compétence provinciale dans le domaine de la santé pour justifier son silence et son inaction ${ }^{40} \%$.

Le comportement de Jean Chrétien rappelle celui de premier ministre Robert Borden pendant la crise scolaire ontarienne. Lorsque le Canada participe à la Première Guerre mondiale, l'unité nationale est sérieusement menacée par les accusations de manque d'empressement des Canadiens français à s'enrôler. En fait, la crise scolaire en Ontario devient un obstacle à l'enrôlement. Pour les nationalistes du Québec et notamment Henri Bourassa, les sacrifices demandés à la population n'ont aucun sens. C'est en Ontario, plutôt qu'en Europe, que le fait français est menacé. Ceci incite Henri Bourassa et des nationalistes à dire "qu'il vaut mieux combattre "les Boches d'Ontario" que d'aller se faire tuer en Europe ${ }^{41}$ ».

C'est dans ce contexte de polarisation des opinions et d'accusation de manque de loyauté envers le Canada et à l'Empire que les députés de la Chambre des communes sont invités à débattre, en mai 1916, du rôle du Parlement fédéral dans la crise scolaire de l'Ontario. Les chances de succès sont bien minces pour l'opposition libérale, dirigée par Wilfrid Laurier, lorsque cette dernière présente une motion invitant « respectueusement l'Assemblée législative de l'Ontario à faire en sorte qu'il ne soit pas porté atteinte au privilège que les enfants 
d'origine française ont de recevoir l'enseignement dans leur langue maternelle ${ }^{42} »$. D'abord le premier ministre conservateur Robert Borden indique aux membres de son parti, lorsque ces derniers l'interpellent, qu'il est hors de question que le gouvernement fédéral s'immisce dans un dossier de responsabilité provinciale ${ }^{43}$. Ensuite, lors du débat sur la motion libérale à la Chambre des communes, Borden réitère sa position constitutionnelle en matière d'éducation ${ }^{44}$. La motion libérale est battue par la majorité conservatrice.

La mobilisation de la population et l'appui du Québec ne suffisent pas à convaincre le gouvernement ontarien de modifier sa politique scolaire tant décriée. Le dénouement de la crise, dans un sens favorable aux intérêts des FrancoOntariens, surviendra graduellement au terme de changements radicaux chez les acteurs ontariens du conflit. Dans le cas de Montfort, ce sont les tribunaux qui donnent raison à la coalition. Puisque l'action militante et les négociations n'ont pas amené la Commission de restructuration des services de santé en Ontario, et encore moins le gouvernement conservateur, à changer leur décision, les dirigeants de l'hôpital Montfort espèrent que les tribunaux appuieront leur cause. La Cour divisionnaire de la Cour supérieure de justice de l'Ontario, en novembre 1999, puis la Cour d'appel de l'Ontario, en décembre 2001, reconnaissent l'hôpital Montfort comme une institution indispensable pour la communauté. Les juges de la Cour d'appel appuient la notion de complétude institutionnelle, c'est-à-dire que la capacité d'un groupe à se doter d'institutions s'inscrit dans le processus d'épanouissement de la collectivité ${ }^{45}$.

\section{Traits valorisés de la mémoire collective}

Les raccourcis historiques, imposés à l'événement du Règlement 17 par les dirigeants de S.O.S. Montfort, consti- 
tuent une reconstruction de l'histoire pour servir les intentions du présent et devenir une référence culturelle. Le cas de l'hôpital Montfort constitue un laboratoire intéressant pour analyser l'imaginaire qui amène un groupe à se mobiliser pour témoigner de sa détermination, pour enrichir et actualiser le contenu de la mémoire collective, ainsi que pour projeter la communauté dans l'avenir. Cette mémoire, espèrent les dirigeants de la coalition, rejoint les aspirations de tous ceux et celles qui font partie du groupe franco-ontarien. D'ailleurs, quatre thèmes dominent leurs discours, notamment ceux de la présidente.

L'idée de l'indispensable lutte, comme caractéristique de l'être franco-ontarien, est omniprésente dans les discours des dirigeants de S.O.S. Montfort et dans certains éditoriaux du Droit. L'évocation du Règlement 17 est destinée à rappeler aux Franco-Ontariens que leur combat pour la reconnaissance de leurs droits est à la fois ancien mais aussi inséparable de leur épanouissement. La lutte est un trait valorisé, qui s'inscrit dans la continuité historique de la communauté. Dès le lendemain de l'annonce de la fermeture de l'hôpital Montfort, la présidente de S.O.S. Montfort affirme que "un fait marquant de la communauté franco-ontarienne, c'est quand elle se sent menacée qu'elle sort tout son enthousiasme, toute sa vitalité ${ }^{46} »$. Lors du rassemblement du 22 mars, Pommainville affirme que les Franco-Ontariens « vont se battre avec courage, audace et détermination et ils vont vain$\mathrm{cre}^{47}$ ». Pour sa part, le président-directeur général et éditeur du Droit, Pierre Bergeron, affirme que le quotidien qu'il dirige " reprend le flambeau de cette lutte incessante contre les forces du rapetissement et du mépris ». Cette lutte, comme il se plaît à le rappeler, caractérise l'être franco-ontarien à l'instar de la devise du quotidien qu'il dirige : "l'avenir est à ceux qui luttent ${ }^{48} »$. L'idée de la lutte est reprise dans le quotidien. À la 
veille du rassemblement, on publie en première page la photo d'un nouveau-né à l'hôpital Montfort avec le titre " Naître au combat». On ajoute " [...] [qu'] il luttera toute sa vie pour vivre en français ${ }^{49} »$.

Cette indispensable lutte devient aussi un signe de la vitalité du groupe franco-ontarien. Le cas de la bataille de Montfort démontre que les Franco-Ontariens sont ni des « dead ducks», ni "des cadavres encore chauds ", expressions utilisées dans certains milieux québécois pour qualifier le fait français hors Québec. Il y a une volonté de démontrer sa vitalité et de combattre le misérabilisme, approche souvent utilisée pour aborder et projeter le fait français en milieu minoritaire. Devant les membres du club Richelieu d'Ottawa, la présidente de S.O.S. Montfort affirme : « Il y a 80 ans, on voulait nous museler [en faisant référence au Règlement 17] mais presque un siècle plus tard, nous sommes toujours là, toujours vivants $^{50} \ldots$.. Lors du rassemblement du 22 mars, elle affirme non sans une certaine fierté : "Les Canadiens regardent aujourd'hui un peuple debout ${ }^{51}$ !»

Le second thème est celui de la solidarité. En évoquant le Règlement 17, les animateurs du mouvement S.O.S. Montfort cherchent à imposer l'unanimité dans les rangs au moment de la mobilisation. Ils craignent la division chez les Franco-Ontariens, car elle pourrait provoquer l'échec. «Il ne faut pas se diviser!", répète le sénateur Jean-Robert Gauthier aux responsables de la coalition ${ }^{52}$.

Un autre thème est celui de l'unanimité et de la difficile acceptation de la dissidence. Lorsqu'un compromis, soit la création d'un pavillon francophone au sein de l'hôpital Civic d'Ottawa, est proposé, la dirigeante de la coalition le condamne, non sans provoquer des vagues. Gisèle Lalonde rejette catégoriquement ce projet, car cela signifierait une trahison pour la communauté. Lors d'une conférence de presse, 
elle s'emploie, pour reprendre ses mots, «à tuer dans l'œuf» l'idée. En agissant ainsi, ses propos constituent une déclaration de guerre à l'égard de ceux qui cherchent une solution autre que le maintien de l'hôpital Montfort. Lalonde compare les francophones qui prônent l'idée d'un pavillon francophone à des collaborateurs, plutôt que des traitres. Avec de tels mots, la dissidence se fait plus discrète ${ }^{53}$. Le sort réservé à la dissidence obéit à des fins stratégiques, soit d'offrir un front uni. Mais cela devient aussi un révélateur de la manière que la coalition conçoit le fonctionnement de la communauté francoontarienne et comment cette dernière devrait se projeter.

Cet effort de chasser la dissidence constitue un cas d'utilisation de l'histoire pour un usage précis, de volonté de s'approprier certains éléments du passé pour influencer le contenu de la mémoire ${ }^{54}$. En réalité, les Franco-Ontariens n'ont pas toujours fait preuve d'unanimité au cours du long épisode de la crise scolaire. Les gens de la région de Sudbury et du sud de la province ont accepté le Règlement 17 en envoyant leurs enfants dans les écoles publiques pourtant décriées. En fait, c'est surtout dans la région d'Ottawa et dans Prescott-Russell que la résistance au Règlement 17 est la mieux organisée et la plus médiatisée ${ }^{55}$. L'unité des Franco-Ontariens est donc un souvenir embelli lorsqu'on la confronte aux faits historiques.

Le dernier thème est celui de la définition même de la communauté : de canadienne-française à franco-ontarienne. Les dirigeants de la coalition n'insistent pas trop sur cet aspect mais on constate un déclin ou plutôt la quasi-absence de la référence canadienne-française. L'épisode de l'établissement hospitalier Montfort témoigne de la métamorphose de la mémoire collective et de l'imaginaire d'un groupe qui, à l'époque du Règlement 17, s'inscrit dans celui de la nation canadienne-française. Il reflète aussi la construction d'une 
identité collective qui serait en deuil d'une nation, puisque la nation canadienne-française s'est à ce point transformée dans les années 1960 qu'il est possible d'affirmer qu'elle a cessé d'exister ${ }^{56}$.

\section{$* * *$}

La construction de la communauté est un processus social. En s'inspirant du concept de nation imaginée de Benedict Anderson ${ }^{57}$, le cas Montfort aide à analyser les efforts pour définir, mobiliser et actualiser le contenu de la mémoire collective des Franco-Ontariens. Ces derniers possèdent les attributs d'un "groupement imaginé ». En effet, il y a des efforts pour créer une mémoire et l'entretenir pour donner du sens à l'idée d'appartenance à la communauté franco-ontarienne. En privilégiant certains symboles, références, comportements, pratiques culturelles, on souhaite que ce sens d'appartenance devienne des traits d'identification pour l'ensemble le plus vaste possible des membres de cette communauté.

En jetant dans le mêlée un événement historique particulier, la crise engendrée par le Règlement 17, les membres de la coalition S.O.S. Montfort n'hésitent pas à épurer l'histoire de cette crise afin de n'en retenir que les éléments les plus spectaculaires, pour ainsi frapper l'imagination et proposer une lecture de la situation actuelle. En rappelant le Règlement 17, il y a un désir, de la part des responsables de la coalition, de faire référence à une expérience historique dont le souvenir serait commun à tous les membres de la communauté pour ainsi mobiliser et renforcir la cohésion du groupe. Cette référence est importante puisqu'elle met de la chair autour d'une identité dont la définition ne se limite pas qu'à la valorisation d'une langue et de pratiques culturelles distinctes. 
L'étude des mémoires collectives révèle cette tendance à gommer les conflits sociaux et à proposer une vision unanimiste. L'événement retenu est simplifié pour faciliter son utilisation et le rendre performant dans les stratégies de mobilisation. À quoi bon s'empêtrer dans ce qui est perçu comme des détails (le contexte de la crise scolaire, les acteurs et les stratégies utilisées) alors que l'évocation du Règlement 17 suffit à frapper l'imaginaire. Après tout, le Règlement 17 rappelle l'existence d'un sentiment d'hostilité au fait français en Ontario, d'un mouvement de mobilisation ou plutôt d'une levée en masse de la collectivité, d'un courage et d'une détermination (thème de la lutte); mais aussi d'une victoire. Voilà ce que l'on souhaite que les gens retiennent de l'événement et non pas sa complexité. Perçus comme des raccourcis par les historiens, les usages du Règlement 17 par S.O.S. Montfort reflètent ce processus d'évocation d'un événement historique et la légitimité qu'il procure à la formation de la communauté.

\section{NOTES}

${ }^{1}$ Je remercie André Larose et les évaluateurs anonymes de la revue pour leurs commentaires et suggestions.

${ }^{2}$ Pierre Jury et Denis Gratton, « Solidarité », Le Droit, 26 février 1997, p. A-2. Voir aussi l'éditorial de Pierre Bergeron, «La gifle », Le Droit, 26 février 1997, p. A-18.

${ }^{3}$ Béatrice Richard, La mémoire de Dieppe. Radioscopie d'un mythe, Montréal, VLB Éditeur, 2002, pp. 24-25. Voir aussi Charles Minguet, « Mythes fondateurs chez Bolívar : quelques aspects ", Cabiers des Amériques latines, n 29-30 (janvier-décembre 1984), pp. 135-142; Antoine Sassine, « Symboles de la dualité identitaire canadienne ", Études canadiennes / Canadian Studies, n 41 (1996), pp. 145-155; Traki Zannad, « Mémoire collective », Les frontières de l'imaginaire. Cabiers de l'imaginaire. Paris, L'Harmattan, 1993, pp. 91-98. 
${ }^{4} \mathrm{Au}$ cours des dernières années, l'étude de la mémoire collective et surtout de son utilisation a donné lieu à une production intéressante au Canada. Signalons les études de Patrice Groulx, Pièges de la mémoire. Dollard des Ormeaux, les Amérindiens et nous, Hull, Vents d'Ouest, 1998, 436 p. ; Henry Vivian Nelles, The Art of Nation-Building: Pageantry and Spectacle at Quebec's Tercentenary, Toronto, University of Toronto Press, 1999, 397 p., ou encore Alan Gordon, Making Public Pasts. The Contested Terrain of Montreal's Public Memories, 1891 1930, Montréal et Kingston, McGill-Queen's University Press, 2001, 233 p. À cette courte liste ajoutons l'ouvrage de Jonathan Vance sur les efforts et le devoir de mémoire après la Première Guerre mondiale intitulé Death So Noble: Memory, Meaning and The First World War, Vancouver, University of British Columbia Press, 1997, 319 p., et celui de Colin M. Coates et Cecilia Morgan, Heroines and History: Representations of Madeleine de Verchères and Laura Secord, Toronto, University of Toronto Press, 2002, 368 p.

${ }^{5}$ Sacha Richard, « Commémoration et idéologie nationale en Acadie. Les fêtes du bicentenaire de la déportation acadienne ", Mens - Revue d'bistoire intellectuelle de l'Amérique française, vol. III, $\mathrm{n}^{\circ} 1$ (automne 2002), p. 30.

'Jenéa Tallentire, «Strategies of Memory: History, Social Memory, and the Community ", Histoire sociale / Social History, vol. 34, $\mathrm{n}^{\circ} 67$ (mai 2001), p. 206.

${ }^{7}$ Raoul Girardet, Mythes et mythologies politiques. Paris, Seuil, 1986, p. 13.

${ }^{8}$ Ibid.

${ }^{9}$ Bergeron, «La gifle », loc. cit, p. A-18.

${ }^{10}$ Jean-Michel Gauthier, «Le cri de Montfort, d'un océan à l'autre », Le Droit, 23 mars 1997, p. A-10.

${ }^{11}$ Page publicitaire payée par l'ACFO, Le Droit, 23 mars 1997, p. A-27.

${ }^{12}$ Alan Cairns, Constitution, Government and Society in Canada: Selected Essays, Toronto, McClelland and Stewart, 1988, 304 p. ; Charter versus Federalism: The Dilemmas of Constitutional Reform, Montréal, McGill-Queen's University Press, $1992,150 \mathrm{p}$.

${ }^{13}$ Gisèle Lalonde, Jusqu'au bout !, Ottawa, Le Nordir, 2003, p. 265.

${ }^{14}$ Ibid., p. 264.

${ }^{15}$ Ibid., p. 255.

${ }^{16}$ Sur les débuts de l'hôpital Montfort, voir Roxanne Deevey, Montfort et Le Droit : même combat? Objectivité journalistique et militantisme communautaire, Ottawa, Le Nordir, 2003, pp. 28-33. 
${ }^{17} \mathrm{Je}$ m'inspire des catégories de lieux de mémoire de Llobera. Josep R. Llobera, "The Role of Historical Memory in Catalan National Identity ", Social Antbropology, vol. 6, n 3 (1998), p. 333.

${ }^{18}$ Lalonde, op. cit., p. 278. La présentation de la lutte pour garder l'hôpital Montfort comme un enjeu national domine aussi la couverture de cette bataille par le quotidien Le Droit. Voir à ce sujet Deevey, op. cit., pp. 53-54.

${ }^{19}$ Lalonde, op. cit, p. 267.

${ }^{20}$ Ibid., p. 264.

${ }^{21}$ Charles Burroughs, "Tout est politique ", Le Droit, 23 mars 1997, p. A 7.

${ }^{22}$ Jean R. Burnet et Howard Palmer, "Coming Canadians ». An Introduction to a History of Canada's Peoples. Toronto, McClelland and Stewart, 1988, p. 40.

${ }^{23}$ Jacques Paul Couturier en collaboration avec Wendy Johnston et Réjean Ouellette, Un passé composé. Le Canada de 1850 à nos jours, Moncton, Éditions d'Acadie, 1996, pp. 102-103.

${ }^{24}$ Margaret Prang, "Clerics, Politicians and the Bilingual Schools Issue in Ontario, 1910-1917 ", dans Craig Brown, dir., Minorities, Schools and Politics. Essays. Toronto, University of Toronto Press, 1969, p. 92.

${ }^{25}$ Pierre Savard, «Relations avec le Québec », dans Cornelius J. Jaenen, dir., Les Franco-Ontariens, Ottawa, Presses de l'Université d'Ottawa, 1993, p. 233.

${ }^{26}$ Robert Choquette, Langue et religion. Histoire des conflits anglo-frangais en Ontario, Ottawa, Les Éditions de l'Université d'Ottawa, 1980 [1977], $2^{\mathrm{e}}$ édition, p. 172.

${ }^{27}$ Ibid., p. 170.

${ }^{28}$ Chad Gaffield, Aux origines de lidentité franco-ontarienne. Éducation, culture et économie, Ottawa, Presses de l'Université d'Ottawa, 1993, traduction de Gilles Hénault avec la collaboration de Lise Demers, 284 p.

${ }^{29}$ Lalonde, op. cit., p. 264.

${ }^{30}$ Bergeron, « Debout », Le Droit, 23 mars 1997, p. A-9. Pour une critique de la couverture de la lutte pour conserver l'hôpital Montfort par ce quotidien francophone, voir Deevey, op. cit.

${ }^{31}$ Jean Hamelin et Nicole Gagnon, Histoire du catholicisme québécois. Le XXe siècle, tome 1, 1898-1940, Montréal, Boréal Express, 1984, p. 98.

${ }^{32}$ Ibid. 
${ }^{33}$ Choquette, op. cit., p. 217.

${ }^{34}$ Michel Gratton, Montfort. La lutte d'un peuple, Ottawa, Centre franco-ontarien de ressources pédagogiques, 2003, pp. 27-30.

${ }^{35}$ "Les discours ", Le Droit, 23 mars 1997, p. A-5.

${ }^{36}$ Suzanne Dansereau, "Missions communes en vue ", Le Devoir, 8 mars 1997, p. A-8. Voir aussi M. Gratton, op. cit., pp. 63, 68.

${ }^{37}$ Cité dans Gratton, op. cit., p. 63.

${ }^{38}$ Choquette, op. cit, p. 190.

${ }^{39}$ Bernard L. Vigod, Quebec Before Duplessis. The Political Career of Louis-Alexandre Taschereau, Kingston et Montréal, McGill-Queen's University Press, 1986, pp. 124-125.

${ }^{40}$ Cité dans Gratton, op. cit., p. 66.

${ }^{41}$ Réal Bélanger, Wilfrid Laurier. Quand la politique devient passion. Québec et Montréal, Presses de l'Université Laval et Entreprises Radio-Canada, 1986, p. 419 ; Peter Oliver, « The Resolution of the Ontario Bilingual Schools Crisis, 1919-1929 ", Joumal of Canadian Studies / Revue d'études canadiennes, volume 7, février 1972, pp. 22-45.

${ }^{42}$ Bélanger, op. cit., p. 421.

${ }^{43}$ Prang, loc. cit., pp. 85-111.

${ }^{44}$ Robert Laid Borden, Memoirs, présentées par Henry Borden, Toronto, MacMillan Company, 1938, volume 2, pp. 590-591.

${ }^{45}$ Deevey, op. cit., pp. 32-33.

${ }^{46}$ Jury et Gratton, loc. cit., p. 2.

${ }^{47}$ J.-M. Gauthier, « Le cri de Montfort, d'un océan à l'autre », Le Droit, 23 mars 1997, p. A-10.

${ }^{48}$ "Les discours », Le Droit, 23 mars 1997, p. A-5.

${ }^{49}$ Annie Morin, « Naître au combat », Le Droit, 22 mars 1997, p. 1.

${ }^{50}$ Jules Richer, « La fermeture donnerait des munitions aux "fervents de la séparation" ", Le Devoir, 6 mars 1997, p. A-4.

${ }^{51}$ Cité dans Gratton, op. cit., p. 106.

${ }^{52}$ Cité dans Lalonde, op. cit., p. 287. 
${ }^{53}$ Gisèle Lalonde revient sur cet épisode dans son autobiographie. Elle reconnaît que l'usage du mot collaborateur était « une déclaration troublante ». Ibid., pp. 284-285.

${ }^{54}$ Pierre Nora écrit au sujet de la mémoire : « Parce qu'elle est affective et magique, la mémoire ne s'accommode que de détails qui la confortent; elle se nourrit de souvenirs flous, télescopants, globaux ou flottants, particuliers ou symboliques, sensible à tous les transferts, écrans, censure ou projections ». Pierre Nora, dir., Les lieux de mémoire, Paris, Gallimard, 1984, p. xix.

${ }^{55}$ Oliver, loc. cit. ; Jacques Taillefer, « L'ACFEO à Sudbury, 1910-1927 », dans Guy Gaudreau, dir., Bâtir sur le roc. De l'ACFÉO à l'ACFO du Grand Sudbury (1910-1987), Sudbury, Prise de parole, 1994.

${ }^{56}$ Plusieurs ouvrages ont été publiés sur cette question. Le lecteur peut consulter Michel Bock, Quand la nation débordait les frontières : les minorités françaises dans la pensée de Lionel Groulx, Montréal, Hurtubise HMH, 2004, 452 p. ; Yves Frenette, avec la collaboration de Martin Pâquet, Brève bistoire des Canadiens français, Montréal, Boréal, 1998, 211 p. ; Marcel Martel, Le Deuil d'un pays imaginé. Rêves, luttes et déroute du Canada français. Les relations entre le Québec et la francophonie canadienne, Ottawa, Les Presses de l'Université d'Ottawa, 1997, 203 p. ; Joseph Yvon Thériault, dir., Francophonies minoritaires au Canada. L'état des lieux, Moncton, Édition d'Acadie, 1999, 578 p.

${ }^{57}$ Benedict Anderson, Imagined Communities: Reflections on the Origin and Spread of Nationalism, Londres, Verso Editions / NLB, 1983, 160 p. 